\title{
Reduced Symmetrizer Equation
}

\section{By R. Purushothaman Nair}

Vikram, Sarabhai Space Centre(VSSC)

Abstract- This paper revisits the real symmetrizer equation in the literature to transform it into a reduced symmetrizer equation. This reduction can be accomplished by decomposing the symmetrizer of the equation. The reduced equation has a diagonal matrix as its symmetrizer and can be further decomposed into more such equations. These reduced equations are coexisting and synchronized with the original symmetrizer equation. Associated results concerning the reduced symmetrizer equation are introduced. A numerical algorithm for symmetrizer computation is developed based on these results. Typical symmetrizer problems in the literature are solved using the algorithm and the results are presented.

Keywords: symmetric matrix factorization, symmetrizer equation, symmetrizer, similarity symmetrization, diagonal matrix symmetrizer.

GJSFR-F Classification: MSC 2010: 15A21, 15A23, 65J10

Strictly as per the compliance and regulations of:

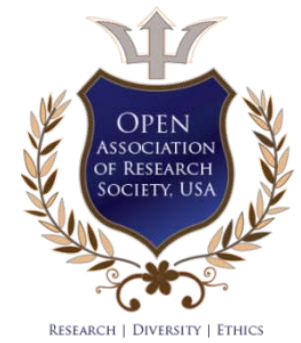

(C) 2020. R. Purushothaman Nair. This is a research/review paper, distributed under the terms of the Creative Commons Attribution-Noncommercial 3.0 Unported License http://creativecommons.org /licenses/by-nc/3.0/), permitting all non commercial use, distribution, and reproduction in any medium, provided the original work is properly cited. 

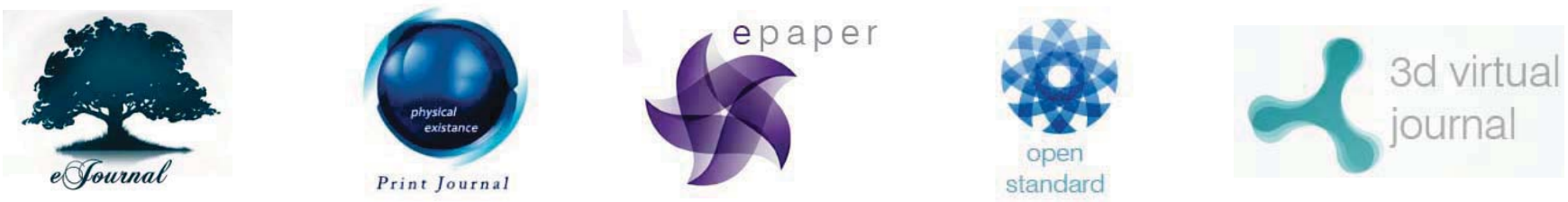

\title{
Reduced Symmetrizer Equation
}

\author{
R. Purushothaman Nair
}

Abstract-This paper revisits the real symmetrizer equation in the literature to transform it into a reduced symmetrizer equation. This reduction can be accomplished by decomposing the symmetrizer of the equation. The reduced equation has a diagonal matrix as its symmetrizer and can be further decomposed into more such equations. These reduced equations are coexisting and synchronized with the original symmetrizer equation. Associated results concerning the reduced symmetrizer equation are introduced. A numerical algorithm for symmetrizer computation is developed based on these results. Typical symmetrizer problems in the literature are solved using the algorithm and the results are presented.

Keywords: symmetric matrix factorization, symmetrizer equation, symmetrizer, similarity symmetrization, diagonal matrix symmetrizer.

\section{INTRODUCTION}

F.G Frobenius [6] introduced symmetric factorization of matrices in 1910. Frobenius finding was that any given matrix $A \in R^{n \times n}$ can be factorized as $A=S_{1} S_{2} ; S_{1}=S_{1}^{T}, S_{2}=S_{2}^{T} ; S_{1}, S_{2} \in R^{n \times n}$. Frobenius also stated that one among $S_{1}, S_{2}$ or both can be nonsingular. Thus Frobenius hints that there can be left and right symmetrizers for $A$. Here for convenience, we choose $S_{1}$ as nonsingular. Accordingly there exists a matrix $B=S^{-1}$ so that $B A=S_{2}$ is symmetric. The nonsingular symmetric matrix $B$ is called a symmetrizer of $A[1,3,4,6,21]$. In the same year, J. Marty [15] introduced symmetrization of linear integral operators. They were the pioneers who worked in the area of symmetrizing matrices. Their work remained dormant for quite some decades till 1950s. Towards the end of 1950s, matrix symmetrization again drew the attention of many researchers. Notably among them, Professor J.L Howland [8] and his team of researchers, E.J Desautels [3], F.J. Farrel [9] and other mathematicians, Olga Taussky and Hans Zassenhaus [22], Marcus and Khan [14] actively pursued this work of symmetrizing $A$. Marcus and Khan [14] studied in a detailed way, a more general equation $X A=A^{T} X$ where $X$ need not be symmetric and discussed its complete solution. These studies contributed to strengthening many theoretical aspects of symmetrizers. It is reported in Dopico

Author: Advanced Technology Vehicles and Sounding Rockets Project(ATVP)(Rtd.), Vikram, Sarabhai Space Centre(VSSC), ISRO P.O, Thiruvananthapuram-695022, India.

e-mail: rpnprasadam@gmail.com 
and Uhlig [4] that several attempts by many researchers to compute numerically a symmetrizer during 1960s to early half of 1970s landed into instability and were abandoned mid-way. An account of those failed attempts is presented in Uhlig [24]. It was Olga Taussky and Hans Zassenhaus [22] who gave a new definition for the Frobenius equation as $B A=A^{T} B$, or the similarity transformation $B A B^{-1}=A^{T}$. Later Taussky [21] independently developed the necessary and sufficient conditions and associated theories for $A$ to be symmetrized and the role of symmetrizer $B$ in the study of $A$. Due to these contributions, Frobenius equation became known as Taussky's Symmetrizer Equation. Sen and Venkaiah [23] proposed a solution to similarity symmetrization in the context of solving asymmetric control system problems. It is reported in [4] that method proposed in [23] can be used for low dimensional matrices $A$.

Adhikari's [1] work in year 2000 states that symmetrizing associated asymmetric matrices of linear systems will be convenient. We can then extend the application of established analytical tools for symmetric systems to such cases also. Illustrative examples solving dynamic linear systems in this manner are presented in [1]. What Adhikari [1] introduced is termed as equivalence symmetrization. In line with Adhikari, C.Q. Liu [11] introduced an alternative equivalence symmetrization method. Liu also worked on Adhikari's same illustrative examples in [1] for highlighting its easiness and effectiveness.

It is reported in [4] that first successful numerical computation of a symmetrizer was in 2013 by Frank Uhlig [24,25] based on an iterative linear equation solver of Huang and Nong[10]. Recently in 2016, Froilan Dopico and Frank Uhlig [4] successfully came out with more methods of computing symmetrizers using eigen data and linear equations solver.

The motivation behind [1] was that equivalence transformations are the most general class of nonsingular linear transformations achieving more generality and convenience. We here attempt to transform $B A=A^{T} B, A, B \in R^{n \times n}, B=$ $B^{T}, \operatorname{det}(B(1: k, 1: k)) \neq 0, k=1: n, \operatorname{det}(A) \neq 0$ into a reduced symmetrizer equation as $D M=M^{T} D ; D, M \in R^{n \times n}$, where $M$ is similar to $A$ and $D$ is a non singular diagonal matrix. Parter and Youngs [17] considered this topic of symmetrizing $A$ by a diagonal matrix $D \in R^{n \times n}$. Their work was to derive the necessary and sufficient conditions on the existence of $D$ symmetrizing $A$ based on probabilistic rules called conservation and reversibility laws. It was based on George Pimbley's [19] initial value problem for the multi-group transport equation. Pimbley addressed the condition on a non-negative $A \in R^{n \times n}$ that leads to the existence of a positive diagonal matrix $D \in R^{n \times n}$ such that $D A D^{-1}$ is a nonnegative symmetric matrix.

The advantages and specialties of reduced symmetrizer equation are highlighted. Frobenius equation [6] is extended to this reduced symmetrizer equation to decompose it into more such reduced equations. Based on this, we prove that nonderogatory $M$ can be expressed as a linear sum of $n^{2}$ linearly independent matrices having diagonal matrix symmetrizers. A numerical algorithm is developed based on these results for computing a symmetrizer of $A$. This is applied 
for symmetrizing those coefficient matrices of undamped and damped dynamical systems from Adhikari [1]. We also discuss how Frobenius and Taussky's equations are linked to each other.

\section{Reduced Symmetrizer Equation}

Taussky and Zassenhaus [22] and Taussky [21] introduced the conditions of symmetrizability of an asymmetric matrix. According to that, for every $A \in R^{n \times n}$ there is a nonsingular symmetric matrix $S \in R^{n \times n}$ transforming it into $A^{T}$. The conditions are :

i) $A$ is the product of two symmetric matrices, one of which is positive definite.

ii) $A$ is similar to a symmetric matrix.

iii) $A^{T}=S^{-1} A S$ with $S=S^{T} \succ 0$.

iv) $A$ has real characteristic roots and a full set of characteristic vectors.

Taussky's similarity symmetrization of $A$ and conditions are also discussed in $[1,11]$. Symmetrization of $A$ can also be achieved by other type of transformations such as equivalent [1], $\left(A+A^{T}\right) / 2, A A^{T}, A^{T} A$, elementary symmmetrization [20] etc. Adhikari [1] classified the similarity transformation based symmetrization as first kind. He introduced the equivalence transformation based symmetrization and termed it as second kind. This second kind of symmetrization is defined as follows:

v) A matrix $A$ is symmetrizable of the second kind if and only if there exist two nonsingular matrices $L, R$ such that $\tilde{A}=L^{T} A R$ is symmetric.

This is a more general classification. It includes the first kind as a special case, $L^{T}=R^{-1}=R^{-T}$. Let $A \in R^{n \times n}$ be nonsingular as well as all its leading principal submatrices, $A(1: k, 1: k), k=1: n-1$. Recalling from [7], consider elementary reduction, $L^{T} A R=D, L, R=U, D \in R^{n \times n}, L, U$ are unit upper triangular matrices and $D$ is a nonsingular diagonal matrix. Thus it is of the second kind. In a reverse way, this second kind symmetrization can be expressed in terms of diagonalization of $A$ as well. We have $L^{T} A U=D$. Let $B=L L^{T}$. Then $B$ is symmetric positive definite and nonsingular. Consider the nonsingular matrices $S_{1}, S_{2} \in R^{n \times n}$ given by $S_{1}=L B$ and $S_{2}=U B$.

$$
\tilde{A}=S_{1}^{T} A S_{2}
$$

Same $B=L L^{T}$ applied in (1) may be used for simultaneously symmetrizing other coefficient matrices of a given linear system. Now from the definition of a symmetrizer in Frobenious [6], Taussky [21] and Desautels [3], we shall transform the symmetrizer equation using elementary reduction of its symmetrizer.

Let $A \in R^{n \times n}$ be nonsingular. Let it be a symmetrizable matrix as discussed in $[1,6,20]$. Let $B=B^{T} \in R^{n \times n}, \operatorname{det}(B(1: k, 1: k)) \neq 0, k=1: n$ be a symmetrizer of $A$. Recalling from [7], we can decompose $B$ as $L B L^{T}=D$ applying Gauss Elimination (GE) and is unique. Matrices $D, L \in R^{n \times n}$. Here $L$ is a unit lower triangular matrix and $D$ is a nonsingular diagonal matrix. 
We may note that $L B L^{T}=D$ will be stable if $B$ is positive definite by the Cholesky theorem [7]. Recalling from Desautels [3], if $A$ is nonderogatory, $B$ will be diagonalizable as it is congruent to diagonal matrix of eigen values of $A$. Also recall from [7] that if $B$ is column diagonally dominant, we may be able to apply GE without pivoting. If $B$ is symmetric positive definte or semidefinite and ill-conditioned, referring to [7], we may apply permutation matrices for symmetric pivoting and diagonal dominance so that $L\left(P B P^{T}\right) L^{T}=D$. Here $P$ is an appropriate permutation matrix and $D=\operatorname{diag}\left(d_{1}, d_{2}, \ldots, d_{n}\right), d_{i} \geq d_{i+1}, i=$ $1: n-1$.

$$
B A=A^{T} B
$$

Consider $L B L^{T}=D$. We shall rewrite (2) as

$$
L^{-1} D L^{-T} A=A^{T} L^{-1} D L^{-T}
$$

Let $M=L^{-T} A L^{T}$. Then equation (3) can be represented as

$$
D M=M^{T} D .
$$

Lemma 1. Given a symmetrizer $B \in R^{n \times n}, \operatorname{det}(B(1: k, 1: k)), k=1: n \neq 0$ of $A \in R^{n \times n}$, the symmetrizer equation $B A=A^{T} B$ can be transformed into $D M=M^{T} D$ by decomposing $B$ as $L B L^{T}=D, L$ is unit lower triangular, $D$ is diagonal and $M=L^{-T} A L^{T}$.

Proof. The proof follows from (2), (3) and (4). Uniqueness of matrices $L, D$ as a pair follows as the decomposition $L B L^{T}=D$ is unique [7].

We see that $M$ is similar to $A$ and $D$ is congruent to $B$. From (4) it becomes clear that the symmetrizer $B$ should be nonsingular. Frobenius [6] states that in $A=S_{1} S_{2}, S_{1}^{T}=S_{1}, S_{2}^{T}=S_{2}$, either $S_{1}$ or $S_{2}$ can be nonsingular so that $A$ can have a left or right symmetrizer. In (2), we consider the case $B=S_{1}$ as nonsingular for convenience. Equations (3) and (4) prove that symmetrizing $A$ by $B$ is synchronized with symmetrizing $M$ by $D$. This leads us to,

Lemma 2. Consider $B=B^{T} \in R^{n \times n}, \operatorname{det}(B(1: k, 1: k)), k=1: n \neq 0$. Let $L B L^{T}=D, L$ is unit lower triangular and $D$ diagonal. Then $B$ symmetrizes $A \in R^{n \times n}$ iff $D$ symmetrizes $M=L^{-T} A L^{T}$.

Proof. We have

$$
\begin{aligned}
D M=M^{T} D & \Leftrightarrow D L^{-T} A L^{T}=L A^{T} L^{-1} D \\
& \Leftrightarrow L^{-1} D L^{-T} A=A^{T} L^{-1} D L^{-T} \\
& \Leftrightarrow B A=A^{T} B
\end{aligned}
$$

Thus (5) is a necessary and sufficient condition for similarity symmetry. 
Lemma 3. Let $B A=A^{T} B, A, B=B^{T} \in R^{n \times n}, \operatorname{det}(B(1: k, 1: k)), k=$ $1: n \neq 0$ so that $L B L^{T}=D, L$ is unit lower triangular and $D$ is diagonal. Consider $M=L^{-T} A L^{T}=\left(m_{i j}\right) ; i, j=1: n$ where $m_{i j}, m_{j i} \neq 0, j=1: n$. Let $D_{i}=\operatorname{diag}\left(x_{1}, x_{2}, \ldots, x_{n}\right), x_{i} \neq 0, i=1: n$ such that $x_{i} m_{i j}=x_{j} m_{j i}, j=1: n$. Then $D_{i}$ is a symmetrizer of $M$ and $B_{i}=L^{-1} D_{i} L^{-T}$ is a symmetrizer of $A$.

Proof. Let $D=\operatorname{diag}\left(d_{1}, d_{2}, \ldots, d_{n}\right)$. Since column- $i$ and row- $i$ of $M$ are without zeros and from the hypothesis and by Lemma 2, we have

$$
x_{i} m_{i j}=x_{j} m_{j i}, d_{i} m_{i j}=d_{j} m_{j i}, j=1: n .
$$

From (6), we get

$$
\begin{aligned}
x_{i} / x_{j}=d_{i} / d_{j}, j=1: n & \Rightarrow d_{j}=x_{j} d_{i} / x_{i}, j=1: n . \\
& \Rightarrow x_{j}=x_{i} m_{i j} / m_{j i}, j=1: n .
\end{aligned}
$$

Consider any other row- $k$, column- $k$ of $D M$. Applying $d_{j}, j=1: n$ of (7)

$$
\begin{aligned}
d_{k} m_{k j} & =d_{j} m_{j k} \Rightarrow d_{k} / d_{j}=m_{j k} / m_{k j} ; j=1: n \\
\Rightarrow x_{k} / x_{j} & =m_{j k} / m_{k j} \Rightarrow x_{k} m_{k j}=x_{j} m_{j k}, j=1: n, k=1: n .
\end{aligned}
$$

From (8), $D_{i}$ symmetrizes $M$, $\operatorname{det}\left(D_{i}\right) \neq 0$. So $D_{i}$ is a symmetrizer of $M$. Hence by Lemma $2, B_{i}=L^{-1} D_{i} L^{-T}$ is a symmetrizer of $A$.

Corollary 3.1. If $M$ in Lemma 3 has at least one row-i, column-i of non-zero entries, then it has a diagonal matrix symmetrizer $D_{i}$ as derived in (7). This diagonal matrix symmetrizer $D_{i}$ of $M$ is embedded in $M$ itself.

Corollary 3.2. As diagonal matrix $D_{i}$ can be derived from (7) using arbitrary scalar $x=x_{i} \neq 0$, infinitely many symmetrizers of $A$ exist against $L B L^{T}=D$.

Remark 3.1. This result can be used to test the symmetrizability of a given matrix $M$ by a diagonal matrix $D_{i}$ as well as to derive $D_{i}$ using (7). Coupled with Lemma 2 , it can also be used to test the symmetrizability of $A$ by $B$.

Lemma 4. Let $B=B^{T} \in R^{n \times n}, \operatorname{det}(B(1: k, 1: k)), k=1: n \neq 0, L B L^{T}=D$, be a symmetrizer of $A \in R^{n \times n}, L$ unit lower triangular and $D$ diagonal. If $M=L^{-T} A L^{T}$ has a column-i and row-i are without zeros, and $D_{1}, D_{2} \in R^{n \times n}$ are its two diagonal matrix symmetrizers, then they are linearly dependent.

Proof. Let $D_{1}=\operatorname{diag}\left(x_{1}, x_{2}, \ldots, x_{n}\right), D_{2}=\operatorname{diag}\left(y_{1}, y_{2}, \ldots, y_{n}\right)$. Then we have

$$
x_{j}=x_{i} m_{i j} / m_{j i} ; y_{j}=y_{i} m_{i j} / m_{j i} ; j=1,2, \ldots, n
$$

From (9), it follows that $D_{1}=\left(x_{i} / y_{i}\right) D_{2},\left(x_{i} / y_{i}\right) \neq 0$. Hence the result.

Corollary 4.1. If $M$ is without zero entries, then the $n$ symmetrizers $D_{i}$ of $M$ derived from column-i, row-i; $i=1: n$ using (7) will be linearly dependent. 
Corollary 4.2. Let $A \in R^{n \times n}$ and $B_{i} \in R^{n \times n}=L^{-1} D_{i} L^{-T}, i=1: n$ are $n$ diagonalizable symmetrizers of $A$ where $L$ is a unit lower triangular matrix and $D_{i}, i=1: n$ are diagonal matrices. Then $B_{i}, i=1: n$ are linearly dependent.

Proof. Let $M=L^{-T} A L^{T}$. By Lemma $2, D_{i}$ are symmetrizers of $M$. So by Lemma 4 , they are linearly dependent and hence $B_{i}, i=1: n$ are so.

Corollary 4.3. Let $A, B=B^{T} \in R^{n \times n}$. Let $B$ be diagonalizable symmetrizer of $A$ so that $B=L^{-1} D L^{-T}$ and $B A=A^{T} B, L$ a unit lower triangular matrix and $D$ a nonsingular diagonal matrix. Let $D=\sum_{i=1}^{n} \alpha_{i} D_{i}$ where $D_{i}, i=1: n$ constitute a basis of subspace of all diagonal matrices of $R^{n \times n}$. Consider $B=$ $\sum_{i=1}^{n} \alpha_{i} B_{i}$ where $B_{i}=L^{-1} D_{i} L^{-T}, i=1: n$. Then $B_{i}, D_{i}, i=1: n$ are not symmetrizers of $A$ and $M=L^{-T} A L^{T}$ respectively.

Remark 4.1. From Corollary 4.3, we see that in several occasions of handling $B_{i}, i=1: n$, we may miss $L$ but if we use Lemma 3 , this will not happen.

Remark 4.2. Reduced equation $D M=M^{T} D$ is more convenient to handle than $B A=A^{T} B$ and from equation (5), these are inter-dependent. As the decomposition $L B L^{T}=D$ is unique in Lemma $4, M=L^{-T} A L^{T}$ has only one linearly independent diagonal matrix symmetrizer $D$. If $M_{1}, M_{2} \in R^{n \times n}$ are linearly dependent, so do their diagonal matrix symmetrizers, say, $D_{1}, D_{2}$.

We shall extend the Frobenius equation [6] as applicable to diagonal matrix symmetrizers. We have for $D, Z \in R^{n \times n}, D Z D$ is always symmetric where $D$ is a nonsingular diagonal matrix and $Z$ is any symmetric matrix.

Lemma 5. A nonsingular diagonal matrix $D \in R^{n \times n}$ will be a symmetrizer of $M \in R^{n \times n}$ iff there exists a matrix $Z=Z^{T} \in R^{n \times n}$ such that $M=Z D$.

Proof. Suppose $D$ is a symmetrizer of $M$. Then we have

$$
D M=M^{T} D \Leftrightarrow M D^{-1}=D^{-1} M^{T} \Leftrightarrow M=D^{-1} M^{T} D .
$$

In (10), let $Z=D^{-1} M^{T}$ and the result follows. Then $Z$ will be nonsingular if and only if $M$ is so. In $M$, if $r$ columns are dependent then in $Z$, so much rows will be dependent. Conversely, if there is a matrix $Z ; Z=Z^{T}$ such that

$$
M=Z D \Leftrightarrow D M=D Z D
$$

Thus from (11), it is confirmed that $D$ is a symmetrizer of $M$.

Corollary 5.1. As $D M=M^{T} D \Leftrightarrow M=Z D$, let $M=\left(m_{i j}\right), Z=\left(z_{i j}\right) ; i, j=$ $1: n$, then $m_{i j}=m_{j i}=0$ iff $z_{i j}=z_{j i}=0 ; i, j=1: n$.

Corollary 5.2. If $A, B=B^{T} \in R^{n \times n}$, then $B$ will be a symmetrizer of $A$ iff there exists a matrix $S=S^{T} \in R^{n \times n}$ such that $A=S B$.

Remark 5.1 By Corollary 5.1, in $Z, M \in R^{n \times n}$, zeros can be only in identical, symmetric positions $(i, j),(j, i), 1 \leq i, j \leq n$. This result is stochastically termed 
in Parter and Youngs [17] as conservative law. If $m_{i j}=m_{j i}=0$, in every column- $i$, row- $i$ for some $1 \leq i, j \leq n$ of $M$, we see from (11) that there can exist $D$ symmetrizing $M$. In such situations, the test in Lemma 3 is invalid.

Remark 5.2 Consider $Z=D^{-1} M^{T}=M D^{-1}$ in (10). Accounting both the terms, we may assume $Z=\left(D^{-1} M^{T}+M D^{-1}\right) / 2$.

Remark 5.3 Adapting the expression of $Z$ in Remark 5.2 into Frobenius equation [6] and treating $S_{2}=B$ as nonsingular, we have $S_{1}=\left(B^{-1} A^{T}+A B^{-1}\right) / 2$. The singularity of $S_{1}$ is depending on the singularity of $A$. Then we have $A=S_{1} B, B A=B S_{1} B=A^{T} B$. The symmetrizer $B$ has an independent existence and $S_{1}$ is dependent on $A, A^{T}$ and $B$. This is how the Frobenius equation [6] is extended and how the Taussky's and Frobenius equations are linked.

Remark 5.4 We may see how Pimbley's [19] condition is true for non-negative $M, D>0, D M D^{-1}=D Z$ is non-negative and symmetric. From (11), Pimbley's equation [19] can be true if $Z$ is a non-negative diagonal matrix.

Remark 5.5 From (11), it follows that if $Z$ is nonsingular, then $Z^{-1}, Z$ are symmetrizers of $M, M^{T}$ respectively. From Desautels [3], the set of symmetrizers of $M$ is a linear subspace of $R^{n \times n}$ of dimension $n$.

Consider the set of those matrices $M$, symmetrized by $D$ and may be denoted as $D(M)$. We see that cardinality of largest possible set of linearly independent symmetric matrices $Z_{i} ; i=1,2, \ldots \in R^{n \times n}$ which can be applied in $M=Z D$ of $(11)$ is $n(n+1) / 2$. Hence $D$ will be a symmetrizer of matrices $M_{i}=Z_{i} D, i=$ $1: n(n+1) / 2 \in R^{n \times n}$, which are linearly independent. If $D$ symmetrizes $M_{1}, M_{2}$, then it also symmetrizes $\alpha_{1} M_{1}+\alpha_{2} M_{2}$ for nonzero scalars $\alpha_{1}, \alpha_{2}$. So $\alpha_{1} M_{1}+\alpha_{2} M_{2} \in D(M)$. Zero matrix also belongs to $D(M)$. Hence we see that $D(M)$ is a linear subspace of $R^{n \times n}$ of dimension $n(n+1) / 2$. Additionally $I_{n}, M, M^{-1} \in D(M)$. Suppose $Z_{i} ; i=1,2, \ldots n$ are nonsingular and linearly independent set of $n$ diagonal matrices. Then $Z_{i} D ; i=1,2, \ldots, n$ also will be so. This set of diagonal matrices will span the whole subspace of diagonal matrices of $R^{n \times n}$ and is also a subspace of $D(M)$.

Lemma 6. Let $D(M) \subset R^{n \times n}$ denotes the subspace of matrices symmetrized by a diagonal matrix $D \in R^{n \times n}$. If $N \in D(M)$ is nonderogatory, then it can be expressed as a linear sum of $n$ nonsingular matrices $N_{i}, i=1: n \in D(M)$.

Proof. Recall from Desautels $[2,3,13,18]$ that when $N \in R^{n \times n}$ is nonderogatory, it has $n$ simple eigen values as well as $n$ distinct symmetrizers spanning the subspace of symmetrizers of $N$. Any symmetrizer $B$ of $N$ will be congruent to the diagonal matrix of its eigen values and so will be diagonalizable [3]. By Lemma 5 , we have $N=Z D, Z=\alpha_{1} Z_{1}+\alpha_{2} Z_{2}+\ldots+\alpha_{n} Z_{n}$, where $Z_{i}, i=1: n$ are symmetrizers of $N^{T}, \alpha_{i}, i=1: n$ are real scalars, as $N^{T}$ as well is nonderogatory and as $Z N^{T}=N Z$. Hence $N=\sum_{i=1}^{n} \alpha_{i} N_{i}=\sum_{i=1}^{n} \alpha_{i} Z_{i} D \in D(M)$ and are nonsingular. 
Corollary 6.1. Let $A \in R^{n \times n}$ be a nonderogatory matrix so that $B_{i} \in R^{n \times n}=$ $L_{i}^{-1} D L_{i}^{-T}, i=1: n$ are $n$ diagonalizable and linearly independent symmetrizers of $A$ where $L_{i}, i=1: n$ are distinct unit lower triangular matrices and $D$ is a nonsingular diagonal matrix. Then $M_{i}=L_{i}^{-T} A L_{i}^{T} \in D(M)$ will span $M$.

Remark 6.1 Taking further, the expression $A=S B$ of Corollary 5.2, $A$ itself will be symmetric if and only if $S B=B S$. Such a situation $M=Z D=D Z$ is there only when $Z$ is zero or is a diagonal matrix. Also if $A$ is nonderogatory, we can find exactly $n$ symmetric matrices $S_{i} ; i=1,2, \ldots, n$ and $A=S_{i} B_{i} ; S_{i}=$ $\left(B_{i}^{-1} A^{T}+A B_{i}^{-1}\right) / 2, i=1,2, \ldots, n$. The corresponding scenario with diagonal matrix symmetrizer will be $M_{i}=Z_{i} D_{i} ; i=1,2, \ldots, n$. According to Frobenius[4,6], for a given matrix $A \in R^{n \times n}$, there is a symmetrizer $B \in R^{n \times n}$. But, this is not true with diagonal matrix symmetrization. As in Lemma 5 , only to matrices $M=Z D ; Z=Z^{T}$ have diagonal matrix $D$ as symmetrizer.

Lemma 7. Let $B=B^{T}, \operatorname{det}(B(1, k: 1, k), k=1: n) \neq 0 \in R^{n \times n}$ be a symmetrizer of $A \in R^{n \times n}$. Let $L B L^{T}=D$ where $L$ is unit lower triangular and $D$ diagonal. Consider $M=L^{-T} A L^{T}$ so that $D$ is a symmetrizer of $M$. If $D M$ is diagonalizable, then $D M$ and symmetrizer $B A$ of $A$ are congruent.

Proof. We have $D, D M^{i}, i=1,2, \ldots$ are symmetrizers of $M$. Consider

$$
D M=L_{1}^{-1} D_{1} L_{1}^{-T}
$$

Now by analogy, $L_{1}^{-1} D_{1} L_{1}^{-T}$ is a symmetrizer of $M, D_{1}$ is a symmetrizer of $M_{1}=L_{1}^{-T} M L_{1}^{T}, D_{1} M_{1}$ is a symmetrizer of $M_{1}$. From (12), we have,

$$
L_{1}^{-1} D_{1} L_{1}^{-T} L^{-T} A L^{T}=L A^{T} L^{-1} L_{1}^{-1} D_{1} L_{1}^{-T}
$$

Equation (13) can be rearranged as below.

$$
L^{-1} L_{1}^{-1} D_{1} L_{1}^{-T} L^{-T} A=A^{T} L^{-1} L_{1}^{-1} D_{1} L_{1}^{-T} L^{-T}
$$

Let this derived symmetrizer of $A$ from (14) be $B_{1}$ so that we have

$$
\begin{aligned}
B_{1} & =L^{-1} L_{1}^{-1} D_{1} L_{1}^{-T} L^{-T} \\
& =L^{-1} D M L^{-T}=B A .
\end{aligned}
$$

Remark 7.1 We may generalize this result that if $L B L^{T}=D$, the symmetrizers $D, D M, D M^{2}, \ldots$ of $M$ are congruent to the symmetrizers $B, B A, B A^{2} \ldots$ of $A$. The specialty of the congruence is presented in (15) and is that the matrices $L, L^{T}, L^{-1}, L^{-T}$ are commonly applied for these congruences. The point is that only diagonalization of $B$ is in demand for these congruences. According to 
Desautels [3], Taussky [21], symmetrizers $D, D M, D M^{2}, \ldots D M^{n-1}$ of $M$ span a subspace with dimension $n$ if and only if $M$, to that effect $A$ is nonderogatory.

We shall see when $M$ in Lemma 1 will be symmetric.

Lemma 8. Let for a given matrix $A \in R^{n \times n}, B=B^{T}, \operatorname{det}(B(1: k, 1: k), k=$ $1: n) \neq 0$ be a symmetrizer. Let $L B L^{T}=D, L, D \in R^{n \times n}, L$ unit lower triangular and $D$ diagonal. Then $M=L^{-T} A L^{T}$ will be a symmetric matrix iff the positive definite symmetric matrix $\Lambda=L^{-1} L^{-T}$ is a symmetrizer of $A$.

Proof. If $M=L^{-T} A L^{T}$ is symmetric, then we get

$$
\begin{gathered}
L^{-T} A L^{T}=L A^{T} L^{-1} \Leftrightarrow \\
L^{-1} L^{-T} A=A^{T} L^{-1} L^{-T} .
\end{gathered}
$$

From (16), positive definite symmetric matrix $\Lambda=L^{-1} L^{-T}$ symmetrizes $A$.

\section{Decomposition of Reduced Symmetrizer Equation}

We may break equation (11) further.

Lemma 9. Let $M \in R^{n \times n}$ and $D \in R^{n \times n}$ be a nonsingular diagonal matrix. Consider $N=\left(M+D^{-1} M^{T} D\right) / 2$. Then $N=M$ iff $D$ is a symmetrizer of $M$.

Proof. We have if $N=M$, then $D M=\left(D M+M^{T} D\right) / 2$ and hence $D$ is a symmetrizer of $M$. Conversely, if $D$ is a symmetrizer of $M$, then $D N=$ $\left(D M+M^{T} D\right) / 2=D M$ and so $N=M$.

Remark 9.1 Recall that in (11), $Z=\left(D^{-1} M^{T}+M D^{-1}\right) / 2$ and $N=Z D$. In this context, we may recall from K.Y. Fan and A.J. Hoffman [5] that for $A \in R^{n \times n}, S=\left(A+A^{T}\right) / 2$ is the closest to $A$ of all the symmetric matrices $X \in R^{n \times n}$. Thus

$$
\|A-S\| \leq\|A-X\|
$$

Analogues to (17), if $D$ symmetrizes $M$, and $K$ any other nonsingular diagonal matrix, $D, M, K \in R^{n \times n}$, let $Z=\left(K M+M^{T} K\right) / 2$. Then from Lemma 9,

$$
\begin{gathered}
\left.M-\left(M+M^{T}\right) / 2=(M-Z) / 2+\left(Z-M^{T}\right) / 2\right) \Rightarrow \\
\left\|M-\left(M+D M D^{-1}\right) / 2\right\| \leq\left\|M-\left(K M+M^{T} K\right) / 2\right\|
\end{gathered}
$$

In (18), because of von Neumann's [16] characterization of all unitary invariant norms in $R^{n \times n}$, we have $\|M\|=\left\|M^{T}\right\|,\|(M-Z) / 2\|=\left\|\left(Z-M^{T}\right) / 2\right\|$. From (18) we have the result that among all nonsingular diagonal matrices $K \neq I_{n}$ and as $K \rightarrow D$, the symmetric matrix $\left(N+N^{T}\right) / 2 \rightarrow\left(M+M^{T}\right) / 2$ will be closest to $M$ than any other symmetric matrices $\left(K M+M^{T} K\right) / 2$. 
Analogously $\left\|A-\left(A+B A B^{-1}\right) / 2\right\| \leq\left\|A-\left(A+S A S^{-1}\right) / 2\right\|$ where $B$ is a symmetrizer of $A$ and $S$ is any other symmetric matrix, $A, B, S \in R^{n \times n}$.

Remark 9.2 Let $M=\left(N+D^{-1} N^{T} D\right) / 2, M \in R^{n \times n}$. We have $M=Z D$ and $Z=\left(D^{-1} M^{T}+M D^{-1}\right) / 2=M D^{-1}=\left(D^{-1} N^{T}+N D^{-1}\right) / 2$.

So $M^{T}-N^{T}=D(N-M) D^{-1}$. We have another situation that Pimbley's [19] condition is met when $M^{T}-N^{T}$ is nonnegative and symmetric and it is possible even when $M, N$ are not symmetric.

Lemma 10. Let $D(M) \subset R^{n \times n}$ denotes the subspace of matrices symmetrized by a diagonal matrix $D \in R^{n \times n}$. If $N \in D(M)$ has $n$ simple eigen values, then $N=\sum_{i=1}^{n^{2}} M_{i}, M_{i}=1: n^{2} \in R^{n \times n}$ all having diagonal matrix symmetrizers.

Proof. From Lemma 5, we have $N=Z D$ where $Z=Z^{T} \in R^{n \times n}$ is nonsingular. Now $D=\sum_{i=1}^{n} \alpha_{i} D_{i}, D_{i}, i=1: n \in R^{n \times n}$ are nonsingular and diagonal. Since $N=Z D \in D(M)$, by Lemma $6, N=\sum_{i=1}^{n} \beta_{i} Z_{i} D, Z_{i} D \in$ $D(M) ; i=1:, n$. Here $\alpha_{i}, \beta_{i}, i=1: n$ are nonzero scalars. Hence,

$$
\begin{gathered}
N=\alpha_{1} \beta_{1} Z_{1} D_{1}+\alpha_{1} \beta_{2} Z_{1} D_{2}+\ldots+\alpha_{1} \beta_{n} Z_{1} D_{n} \\
+\alpha_{2} \beta_{1} Z_{2} D_{1}+\alpha_{2} \beta_{2} Z_{2} D_{2}+\ldots+\alpha_{2} \beta_{n} Z_{2} D_{n}+\ldots \\
+\alpha_{n} \beta_{1} Z_{n} D_{1}+\alpha_{n} \beta_{2} Z_{n} D_{2}+\ldots+\alpha_{n} \beta_{n} Z_{n} D_{n} \Rightarrow \\
N=M_{1}+M_{2}+\ldots+M_{k} ; k=n^{2} .
\end{gathered}
$$

Since none of the scalars, $\alpha_{i} \beta_{j}=0, i, j=1: n$ is zero in (19), the $n^{2}$ matrices in (20) are linearly independent. Each matrix $M_{i}$ is nonsingular as it is a product of nonsingular matrices. Also $D_{i} ; i=1: n$ symmetrizes $M_{n(j-1)+i}, i, j=1: n$.

Under certain conditions, converse of the Lemma 10 is also true.

Corollary 10.1. Let $N \in R^{n \times n}$ be the sum of $n^{2}$ linearly independent nonsingular matrices $M_{i}, i=1: n^{2} \in R^{n \times n}$ such that $\left(D_{i}^{-1} M_{j}^{T}+M_{j} D_{i}^{-1}\right) / 2=$ $\left(D_{i}^{-1} M_{n(i-1)+j}^{T}+M_{n(i-1)+j} D_{i}^{-1}\right) / 2 ; i, j=1: n$ where $D_{i}, i=1: n \in R^{n \times n}$ are diagonal matrix symmetrizers of $M_{n(i-1)+j} ; i, j=1: n$. If all the matrices $M_{i}, i=1: n^{2}$ are with $n$ simple eigen values, then $N$ also will be symmetrized by a diagonal matrix $D \in R^{n \times n}$ and it has $n$ simple eigen values.

Proof. From the hypothesis and Lemma 6, if $D_{i}$ symmetrizes $M_{n(i-1)+j}$, let

$$
\begin{aligned}
Z_{j}= & \left(D_{i}^{-1} M_{n(i-1)+j}^{T}+M_{n(i-1)+j} D_{i}^{-1}\right) / 2 \Rightarrow \\
Z_{j} D_{i}= & M_{n(i-1)+j} ; i, j=1: n \Rightarrow \\
N= & Z_{1} D_{1}+Z_{1} D_{2}+\ldots+Z_{1} D_{n}+Z_{2} D_{1}+Z_{2} D_{2}+\ldots+Z_{2} D_{n}+\ldots .+ \\
& Z_{n} D_{1}+Z_{n} D_{2}+\ldots+Z_{n} D_{n} \Rightarrow N=Z D
\end{aligned}
$$


In (21) $Z_{1}+Z_{2}+\ldots+Z_{n}=Z$ and $D_{1}+D_{2}+\ldots .+D_{n}=D$ are assigned. As $Z$ is nonsingular, $Z^{-1}$ is a symmetrizer of $N$. Hence $Z$ will be a symmetrizer of $N^{T}$. From Desautels [3], $N^{T}$ is nonderogatory and hence $N$ is so.

Remark 10.1 The $n^{2}$ symmetrizer equations $D_{i}^{-1} M_{n(i-1)+j}^{T}=M_{n(i-1)+j} D_{i}^{-1} ; i, j=$ $1: n$ in (21), where $D=D_{1}+D_{2}+\ldots D_{n}$ and $Z=Z_{1}+Z_{2}+\ldots+Z_{n} ; Z_{i}=$ $\left(D_{j}^{-1} M_{n(j-1)+i}^{T}+M_{n(j-1)+i} D_{j}^{-1}\right) / 2 ; i, j=1: n$ are synchronized among themselves and with equation (2) as $M=Z D$ and $M$ similar to $A$.

\section{a) Symmetrizer Computation Algorithm}

Recall from Lemma 5 that a nonsingular diagonal matrix $D \in R^{n \times n}$ will be a symmetrizer of $M \in R^{n \times n}$ if and only if $M=Z D, Z \in R^{n \times n}, Z=Z^{T}$. We see that $Z$ will be then a symmetrizer of $M$ if it is nonsingular. In a general way, it may be noted that if we can derive a diagonal matrix $D$ at each step, then $Z=\left(D M+M^{T} D\right) * 0.5$ can be computed as an approximate symmetrizer of $M$ for that step. This $D$ has to be improved upon, in an iterative manner so that $D M$ is arbitrarily close to symmetry. Note that it is not essential to decompose $Z$ or $M$ for deriving $D$ as presented in the algorithm below:

Step-1: Initialize iteration index $\mathrm{k}=1$, row index $\mathrm{r}=3$ and current row index $\mathrm{c}=3$. Initially let $M_{r}(k)=\left(m_{i j}(k)\right) ; i, j=1: n$ is set as a matrix similar to $A$. Set $\epsilon>0$ to an arbitrarily chosen small quantity, say $\epsilon=1.0 e-15$. Initialize matrix $M=A, Z=I_{n}, D=I_{n}$.

Step-2: Consider the principal submatrix $M_{r}(k)(1: 1,1: 1)$. It is the scalar $m_{11}(k)$ and is symmetric. Correspondingly to compose a diagonal matrix $D_{k}=$ $\operatorname{diag}\left(d_{1}(k), d_{2}(k), \ldots d_{n}(k)\right.$ for this step- $k$, we choose $d_{1}(k)=1$. For the principal submatrix $M_{r}(k)(1: 2,1: 2)$ we choose $d_{2}(k)=m_{12}(k) / m_{21}(k)$ so that $M_{r}(k)(1: 2,1: 2)$ is symmetric in $D_{r}(k) M_{r}(k)$.

Step-3: Now consider the principal submatrix $M_{r}(k)(1: r, 1: r)$. There are $r-1$ pair of entries $m_{r j}(k), m_{j r}(k), j=1: r-1$ to be addressed in row- $r$ and column $r$. By Corollary 5.1, if any of the row entries $m_{r j}(k)$ is zero, then corresponding column entry $m_{j r}(k)$ also should be zero and in this case we may consider $d_{r}(k)=1$. If non of these pairs are zeros, As a trail, taking into account the first pair, consider $d_{r}(k)=m_{1 r}(k) / m_{r 1}(k)$. Thus $(\mathrm{r}, 1)$ and $(1, \mathrm{r})$ entries in $D_{r}(k) M_{r}(k)$ are identical. Now the effect of this is computed as $e_{r 1(k)}=\sum_{i=1}^{r} \sum_{j=1}^{r}\left(m_{i j}(k)-m_{j i}(k)\right)^{2}$. This computation of error in symmetry is repeated out of row- $r$ and column- $r$ of $D_{r}(k)^{-1} M_{r}(k)^{T}$ and added together to store as $e_{r 1}(k)$. Repeat this error computations $e_{r i}(k), i=1: r-1$ in a similar way with respect to other pairs by applying $d_{r}(k)=m_{i r}(k) / m_{r i}(k), i=1: r-1$. Among these (r-1) errors, let $e_{r l}(k)$ be the minimum against row- $r$ for iteration- $k$ and correspondingly we choose $d_{r}(k)=m_{l r}(k) / m_{r l}(k)$. 
Step-4: Remaining entries of $D_{r}(k), d_{r+1}(k), d_{r+2}(k), \ldots d_{n}(k)$ we set $d_{i}(k)=$ $1, i=r+1: n$. Compute the approximate symmetrizer for submatrix $M_{r}(k)(1:$ $r, 1: r)$ as $Z_{r}(k)=0.5 *\left(D_{r}(k) M_{r}(k)+M_{r}(k)^{T} D_{r}(k)\right)$. Set column- $i$ and row- $i$ in $Z_{r}(k)$ as that of $I_{n}$ for $i=r+1: n$.

Step-5: If iteration index $k=1$, set $e_{r m i n}=e_{r l}(k)$, otherwise if $e_{r l}(k)<e_{r m i n}$ set set $e_{r m i n}=e_{r l}(k)$. In this case the iteration- $k$ is treated as a success. If $e_{r l}(k)>e_{r m i n}$ it is a failure. If iteration- $k$ is a success, store $M=M_{r}(k), D=$ $D_{r}(k), Z=Z_{r}(k)$. Compute $B=Z D^{-1} Z$ as an approximate symmetrizer of $A$ at iteration- $k$ as $A=Z^{-1} M^{T} Z$.

Step-6: Test whether $e_{r m i n}(k)<\epsilon$. If it is so, check whether current row $c=r$. If it is true, set $c=c+1$. Exchange row- $c$ with row- $r$ and column- $c$ with column- $r$. Loop back for carrying out step-2 through step-6. If $r<c$, set $r=c$. If $c>=n$ and $e_{\text {rmin }}<\epsilon$ stop the process, otherwise set $c=n$, loop back to step-2 and repeat the steps 2 through 6 .

\section{b) Assumptions, Theory and Convergence of the Algorithm}

The target is to compute a symmetrizer $B \in R^{n \times n}$ of a given nonsingular matrix $A \in R^{n \times n}$. $A$ is assumed to be as in $[3,6,20]$.

1. So far, we were using the decomposition $L B L^{T}=D$ for developing the theoretical background, deriving $D$ from $B$ and similar matrix $M$ from $A$. Against this, now both $B, D$ are derived from $M$ itself at each iteration. Reduction of assumed symmetrizers $Z_{r}(k)$ is not essential for the procedure. It is thus more practical by avoiding the usual difficulties of round off or truncation errors, pivoting, permutations, division by zero or singularities etc. faced in such a decomposition. The basic assumption about symmetrizer $Z_{r}(k)$ is that because of the KY Fan and A.J. Hoffman [5] result, it is sufficiently close to the symmetrizer of $M_{r}(k)$ at iteration $k$. The fact is that when $n=3$, for row-3 and column-3, only one of the pairs of entries, $\left(m_{31}(k), m_{13}(k)\right.$ and $\left(m_{32}(k), m_{23}(k)\right)$ remains non-identical at iteration- $k$. In order to carry forward this situation to problems with $n>=4$, we address first it as a problem with $n=3$ and once the error is minimized to the preset and specified tolerance vale $\epsilon>0$, row-3 and column- 3 are exchanged with row- 4 and column- 4 and again treat the problem as if it is of dimension $\mathrm{n}=3$. Once these two levels of minimization with $n=3$ are accomplished, stage is set ready for $n=4$ as implemented in step-6. Now the procedure treats the $4 \times 4$ submatrix of the problem and so on. This way, the $n$-dimensional symmetrization is conducted in a conducive environment so that the computed symmetrizers remain close to any actual existing symmetrizer if any, obeying the result we derived here from [5].

2. In step-3, we individually compute errors for symmmetrizing $M_{r}(k), M_{r}(k)^{T}$ by $D_{r}(k), D_{r}(k)^{-1}$ respectively and add both them as a single error value as $e_{r i}(k), i=1: r-1$. This for the stability of the process. If we depend only one of them, soon $D$ may converge to either zero or infinity. Also note that if $D$ is a symmetrizer of $M$ then $D^{-1}$ symmetrizes $M T$. Thus it is also a theoretical requirement. 
3. When $e_{r \min }<\epsilon$, the matrix is assumed to be a symmetrizer of $M_{r} k$. Corresponding to $r=n, D M$ will be symmetric, $B A$ will be symmetric. The process is terminated as it marks the successful convergence of the procedure.

4. We know from Desautels [3] that if $D$ is a symmetrizer of $M$ so do $D M, D M^{2}, \ldots$ etc. . Hence $Z_{r}(k)=0.5 *\left(D_{r}(k) M_{r}(k)+M_{r}(k)^{T} D_{r}(k)\right)$ will be closer to $D_{r}(k) M_{r}(k)$ as and when the successive computations approach $D M$ at successful iteration- $k=1,2, \ldots$ and converges to it when $e_{r m i n}<\epsilon$.

\section{Numerical Examples}

The numerical strategy explained in Section 3 is implemented in MATLAB. The coefficient matrices cited in Adhikari [1] of dynamic systems associated with equations of motion of undamped linear systems and that of equations of motion describing free vibration of a viscously damped linear system are considered here to test the practical use of the procedure. There are real coefficient matrices with complex eigen system. Dopico and Uhlig [4] prove that if $A$ is diagonalizable then the two eigen vectors corresponding to a pair of complex conjugate eigen values of $A$ can be chosen of complex conjugates of each other. Then the symmetrizer built from such an eigen vector basis will be real. We may note that all these examples from Adhikari [1] are diagonalizable. (Results are rounded to 4 decimals). For all the problems $\epsilon=1.0 e-15$ is fixed.

Example 1: This is the first real asymetric matrix cited by Adhikari which is reported as not satisfying Taussky's condition of symmetrizability.

The matrix is $A=\left(\begin{array}{ccc}1.0 & -2.0 & 1.5 \\ 12.0 & 6.0 & 7.0 \\ -2.0 & 4.0 & 9.0\end{array}\right)$. Its eigen values are

$(1.5543+5.0507 \mathrm{i}, 1.5543-5.0507 \mathrm{i}, 12.8914)$. After 32 iterations, the error reduced to $6.107564 \mathrm{e}-16$, the matrix $M$ similar to $A$ is computed as

$$
M=\left(\begin{array}{ccc}
1.1262 & -2.1628 & 1.1215 \\
11.5416 & 6.4407 & 7.9459 \\
-2.9727 & 3.9469 & 8.4331
\end{array}\right)
$$

Its symmetrizer is $D=\operatorname{diag}(1.0000,-0.1874,-0.3773)$. The symmetrization of $M$ is $D M=\left(\begin{array}{ccc}1.1262 & -2.1628 & 1.1215 \\ -2.1628 & -1.2069 & -1.4890 \\ 1.1215 & -1.4890 & -3.1814\end{array}\right)$. Now the symmetrizer of $A$ is computed as $B=\left(\begin{array}{ccc}-18.1025 & -6.6674 & -1.7944 \\ -6.6674 & -1.9765 & -9.7041 \\ -1.7944 & -9.7041 & -14.1343\end{array}\right)$. Symmetrization of $A$ is 
carried out as $B A=\left(\begin{array}{ccc}-94.5221 & -10.9766 & -89.9748 \\ -10.9766 & -37.3404 & -111.1730 \\ -89.9748 & -111.1730 & -197.8291\end{array}\right)$.

Example 2: Adhikari [1] considers only real square matrices as these are the coefficient matrices in the equations of motion of linear vibrations. In this case of undamped system $X \ddot{x}(t)+Y x(t)=0, t>0$, the coefficient matrices $X$ and $Y$ are real and symmetrizable of first kind. These matrices are taken from Ma and Caughey [12] . We may consider the matrix $X=$ $\left(\begin{array}{ccc}0.5740 & 1.3858 & 1.3858 \\ 0.7070 & 0.7070 & -0.7070 \\ 0.4620 & -0.1914 & -0.1914\end{array}\right)$. Results are presented below:

Eigen Values :-1.1360, 1.6648, 0.5608 ; No. of iterations : 72

Error Value : 8.988429e-16 : Symmetrizer $D=\operatorname{diag}(1.0000,1.5931,3.7701)$

Similar Matrix $M=\left(\begin{array}{ccc}0.5069 & 1.2911 & 1.3981 \\ 0.8104 & 0.7382 & -0.7057 \\ 0.3708 & -0.2982 & -0.1555\end{array}\right)$.

Symmetrization of $M: D M=\left(\begin{array}{ccc}0.5069 & 1.2911 & 1.3981 \\ 1.2911 & 1.1761 & -1.1242 \\ 1.3981 & -1.1242 & -0.5864\end{array}\right)$.

Symmetrizer $B=\left(\begin{array}{lll}3.2023 & 3.7397 & 0.0369 \\ 3.7397 & 6.1330 & 1.2816 \\ 0.0369 & 1.2816 & 1.8605\end{array}\right)$.

Symmetrization of $X: B X=\left(\begin{array}{ccc}4.4991 & 7.0747 & 1.7868 \\ 7.0747 & 9.2732 & 0.6011 \\ 1.7868 & 0.6011 & -1.2111\end{array}\right)$.

Now we are considering coefficient matrices $Y 1, Y 2$ of an undamped system with

complex eigen values where $X$ is same as in example-1. $Y 1=\left(\begin{array}{ccc}1.2044 & -5.4424 & 2.7013 \\ 2.1007 & 0.893 & 0.1894 \\ -1.8393 & 0.8953 & 2.5087\end{array}\right)$.

Eigen values are $1.05192517450474+4.01396879901174$,

$1.05192517450474-4.01396879901174 \mathrm{i}$, and 2.44854965099053. 
No.of Iterations: 21 Error Value : 3.245450e-16.

Similar Matrix $M=\left(\begin{array}{ccc}1.1739 & -5.4028 & 2.8105 \\ 2.0130 & 0.8309 & 0.3480 \\ -2.0081 & 0.6674 & 2.5477\end{array}\right)$.

Symmetrzation of $M, D M=\left(\begin{array}{ccc}1.1739 & -5.4028 & 2.8105 \\ -5.4028 & -2.2300 & -0.9341 \\ 2.8105 & -0.9341 & -3.5657\end{array}\right)$.

Symmetrizer $B=\left(\begin{array}{ccc}0.9933 & -0.0510 & -0.1018 \\ -0.0510 & -2.6624 & -0.0623 \\ -0.1018 & -0.0623 & -1.4524\end{array}\right)$.

Symmetrzation of $Y 1$ as $B Y 1=\left(\begin{array}{ccc}1.2764 & -5.5399 & 2.4180 \\ -5.5399 & -2.0126 & -0.7984 \\ 2.4180 & -0.7984 & -3.9306\end{array}\right)$.

Next exmple is Matirx $Y 2=\left(\begin{array}{ccc}0.1995 & 1.8857 & -3.2199 \\ -2.1312 & -0.2236 & 0.3609 \\ 1.0378 & 1.9501 & -1.5606\end{array}\right)$ with coeffi-

cient matrix $X$ same as in example-1. The solution is as follows.

Eigen values: $-1.20087894725344+2.67720413881095 i$,

$-1.20087894725344-2.67720413881095 i$ and 0.813057894506878 .

No.of iterations : 14. Error Value : 2.582360e-16.

Diagonal Matrix Symmetrizer $D:=\operatorname{diag}(1.0000,1.7457,-1.9129)$.

Similar Matrix $M=\left(\begin{array}{lll}1.6391 & 1.7133 & -5.6672 \\ 0.9814 & 1.1319 & -1.3944 \\ 2.9626 & 1.2725 & -4.3597\end{array}\right)$.

Symmetrzing M is $D M=\left(\begin{array}{ccc}1.6391 & 1.7133 & -5.6672 \\ 1.7133 & 1.9760 & -2.4341 \\ -5.6672 & -2.4341 & 8.3398\end{array}\right)$.

Symmetrizer $B=\left(\begin{array}{ccc}-0.6605 & -6.2810 & 0.2562 \\ -6.2810 & 1.0501 & 3.9742 \\ 0.2562 & 3.9742 & 7.5928\end{array}\right)$.

Symmetrzing Y2 is $B Y 2=\left(\begin{array}{ccc}13.5229 & 0.6586 & -0.5400 \\ 0.6586 & -4.3288 & 14.4011 \\ -0.5400 & 14.4011 & -11.2399\end{array}\right)$. 
Example 3: Equations of motion describing free vibration of a viscously damped linear system can be expressed as $X \ddot{x}(t)+Y \ddot{x}(t)+W x(t)=0, t>0$. The matrices $X, Y, W$ are from $R^{n \times n}$. When $X, Y, W$ are symmetric and positive definite, they represent the mass, stiffness, and viscous damping matrices. The scope of this work is limited to demonstrate the individual symmetrization of these coefficient matrices. In this respect, matrix cited in Adhikari [1] in place of $W$ is considered. The matrix $W$ from a damped system claimed to be not symmetrizable of first kind has complex eigen values.

$$
\text { The matrix is } W=\left(\begin{array}{ccc}
4.3160 & -2.5771 & -1.4626 \\
2.7122 & 1.8365 & -0.1999 \\
1.3827 & -2.5631 & 4.3419
\end{array}\right) \text {. }
$$

But it is successfully solved using the decomposition method presented here and the results are presented below.

Eigen Values:2.7938+2.8137i, 2.7938-2.8137i 4.9068;No. of iterations:44

Error :4.286294e-16 ; Symmetrizer $D=\operatorname{diag}(1.0000,-0.8916,-1.1189)$

Similar Matrix $M=\left(\begin{array}{ccc}4.3537 & -2.4003 & -2.1766 \\ 2.6922 & 2.0645 & -1.8430 \\ 1.9452 & -1.4686 & 4.0761\end{array}\right)$.

Symmetrization of $M: D M=\left(\begin{array}{ccc}4.3537 & -2.4003 & -2.1766 \\ -2.4003 & -1.8407 & 1.6432 \\ -2.1766 & 1.6432 & -4.5609\end{array}\right)$.

Symmetrizer $B=\left(\begin{array}{ccc}5.9204 & -16.2577 & -0.7220 \\ -16.2577 & 7.8400 & 4.0794 \\ -0.7220 & 4.0794 & -11.9274\end{array}\right)$.

Symmetrization of $W: B W=\left(\begin{array}{ccc}-19.5403 & -43.2641 & -8.5442 \\ -43.2641 & 45.8402 & 39.9236 \\ -8.5442 & 39.9236 & -51.5470\end{array}\right)$.

\section{Conclusions}

Frobenius [6] or Taussky's real symmetrizer equation [21] can be transformed into a reduced symmetrizer equation (Lemma 1) when the symmetrizer of the given equation is diagonalizable in elementary terms. This reduced equation has a diagonal matrix as its symmetrizer. It is congruent to the symmetrizer of Taussky's equation. It symmetrizes a matrix similar to that of the original equation. It is proved that diagonal matrix symmetrizer is embedded in the concerned matrix itself. This property can be used as a test for diagonal matrix symmetrization (Lemma 3). The reduced symmetrizer equation can be further decomposed into more such reduced equations as proved in Corollary 10.1. 
We conclude that symmetrization in $R^{n \times n}$ is mutually synchronized with symmetrization by diagonal matrices whenever the symmetrizer is diagonalizable. This provides necessary and sufficient conditions for a matrix to be symmetrized using Taussky's equation(Lemma 2) as well as new approaches in solving it. The second symmetric matrix factor of the Frobenius equation, which need not be a symmetrizer, is expressed here in terms of the asymmetric matrix, its transpose and the symmetrizer (Lemma 5). This is the matrix that bridges the Frobenius and Taussky's equations. It is attempted to explain or adapt certain results in [5], [16], [17] and [19] using reduced symmetrizer equation. A symmetrizer computing algorithm is introduced. It is applied on some practical problems in the literature and results are presented.

\section{ACKnOWLedgements}

Author expresses sincere and deep gratitude to the reviewers of this work for their constructive and enlightning feedbacks. He would also like to expresse profound thanks to Dr. G Madhavan Nair, former Chairman, ISRO and Shri PG Ravindran, former Group Head, ATFG/VSSC for the inspring support and encouragments provided. This research did not receive any specific grant from funding agencies in the public, commercial, or not-for-profit sectors.

\section{References Références Referencias}

1. S. Adhikari, On symmetrizable systems of second kind, Journal of Applied Mechanics 67 (7) (2000) 797-802, doi:10.1115/1.1322038.

2. R. Bellman, Introduction to Matrix Analysis, McGraw-Hill, New York, 1960.

3. E. J. Desautels, Symmetrizing matrices, Master's thesis, University of Ottawa (1968). URL http://www.ruor. uottawa.ca/bitstream/10393/10879/1/EC52206.PDF

4. F. Dopico, F. Uhlig, Computing matrix symmetrizers, part 2: New methods using eigendata and linear means; a comparison, Linear Algebra and Its Applications 504 (2016) 590-622, doi:10.1016/j.laa.2015.06.031.

5. K. Y. Fan, A. J. Hoffman, Some metric inequalities in the space of matrices, Proceedings of the American Mathematical Society 6 (1955) 111-116.

6. F. G. Frobenius, U ber die mit einer matrix vertauschbaren matrizen, Gesammelte Abhandlungen, Band 3, Springer (1968) 415-427; and in Sitzungsberichte der Koniglich Preuischen Akademie der Wissenschaften, zu Berlin (1910),115.

7. G. H. Golub, C. F. V. Loan, Matrix Computations, 4th Edition, The Johns Hopkins University Press, Baltimore, Maryland, 1985. 
8. J. L. Howland, A method for computing the real roots of a determinental equation, American Math. Monthly 68 (1961) $235-239$.

9. J. L. Howland, F. J. Farrel, Matrix symmetrizing methods for the algebraic eigenvalue problem, ACM National Conference, Colorado, 1963.

10. J. Huang, L. Nong, An iterative algorithm for solving finitedimensional linear operator equations $\mathrm{T}(\mathrm{x})=\mathrm{f}$ with applications, Linear Algebra and Its Applications 432 (2010) 1176-1188.

11. C. Q. Liu, A method of symmetrization of asymmetrical dynamical systems, Shock and Vibration 12 (2005) 309-315, doi:10.1155/2005/390317.

12. F. Ma, T. K. Caughey, Analysis of linear conservative vibrations, ASME J. Mech. 62 (1995) 685-691.

13. M. Marcus, Basic Theorems in Matrix Theory, 4th Edition, U.S. Dept. of Commerce, National Bureau of Standards, Washington, 1960.

14. M. Marcus, N. A. Khan, On a commutator result of taussky and zassenhaus, Pac. J. Math. 10 (1960) 1337-1346.

15. J. Marty, Existence de solutions singulieres pour certaines Equations de Fredholm, Comptes Rendues 150 (1910) 1031-1033.

16. J. von Neumann, Some matrix-inequalities and metrization of matric-space, Tomsk Univ. Rev. 1 (1937) 286-300.

17. S. V. Parter, J. W. T. Youngs, The symmetrization of matrices by diagonal matrices, Journal of Mathematical Analysis Applications 4 (1962) 102-110.

18. S. Perlis, Theory of Matrices, Addison-Wesley, Massachusetts., 1952.

19. H. G. Pimbley, Solution of an initial value problem for the multi-velocity neutron transport equation with a slab geometry, J. Math. and Mech. 8(1959) 837-866.

20. R. P. Nair, Elementary uniform matrix symmetrization, Journal of Mathematics and Statistical Science. 6 (2020) 140-156.

21. O. Taussky, The role of symmetric matrices in the study of general matrices, Linear Algebra and Its Applications 5 (1972) 147-154, doi: 10.1016/0024-3795(72)90024-9.

22. O. Taussky, H. Zassenhaus, On the similarity transformation between a matrix and its transpose, Pac. J. Math 9 (3) (1959) 893-896, doi:10.2140/pjm.1959.9.893.

23. S. K. Sen, V. C. H. Venkaiah, On symmetrizing a matrix, Indian J. Pure Appl. Math. 19 (6) (1988) 554-561.

24. F. Uhlig, Computing matrix symmetrizers, finally possible via the Huang and Nong algorithm, Linear and Multilinear

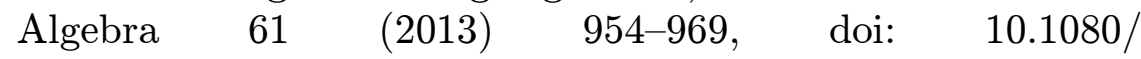
03081087.2012.716427. 
25. F. Uhlig, Method(1) matlab m-file symmorthlongv.m (2012). URL http://www.auburn.edu/•uhligfd/m-files/MatrixSymm/ symmorthlongv.m

$[1,2,3,4,5,6,7,8,9,10,11,12,13,14,15,16,17,18,19,20$, $21,22,23,24,25]$.

Notes 